\title{
Hospital resource utilization associated with pediatric gastrostomy devices
}

\author{
Morgan K Richards ${ }^{* 1}$, Jarod P McAteer ${ }^{1}$, Meera Kotagal ${ }^{2}$, Dennis W Shaw ${ }^{1}$, Ghassan T Wahbeh ${ }^{1}$, Lilah Melzer ${ }^{1}$, \\ Adam B Goldin ${ }^{1}$ \\ ${ }^{1}$ Seattle Children's Hospital, Seattle, Washington, United States \\ ${ }^{2}$ University of Washington Medical Center, Seattle, Washington, United States
}

Received: March 11, 2015

DOI: $10.5430 /$ jha.v4n4p8
Accepted: April 14, 2015

URL: http://dx.doi.org/10.5430/jha.v4n4p8

\begin{abstract}
Objective: Pediatric gastrostomy devices (GD) are associated with frequent planned and unplanned ongoing care. The objective was to examine the association between planned or unplanned repeat intervention and 1) the type of tube at initial placement and 2) the method of initial device placement: operative, endoscopic or image-guided.

Methods: We performed a retrospective review from January 1, 2010 - December 31, 2011 of consecutive patients that received a GD or gastrojejunostomy device (GJ) at a freestanding pediatric hospital. We estimated the risk of hospital utilization in terms of repeat interventions or device-related emergency department visits for initial GJ versus GD placement and based on method of initial device placement.

Results: Over the two year period, 275 (92.0\%) GDs and 24 (8.0\%) GJs were placed. One hundred forty-five (48.5\%) were placed surgically, $113(37.8 \%)$ endoscopically, and 41 (13.7\%) with an image-guided technique. Repeat utilization occurred in $60.9 \%$ of patients. Multivariate Poisson regression estimated that GJs compared to GDs were 2.05 (95\% CI: 1.01 - 4.16) times as likely to have repeat hospital utilization. Multivariate Cox regression estimated that endoscopic and image-guided placements were associated with higher rates of hospital utilization than surgical placement (HR 2.56; 95\% CI: 1.73 - 3.79 and 2.07; $95 \%$ CI: 1.20 - 3.58, respectively).

Conclusions: Resource utilization after GD/GJ placement is not equal relative to the method of initial placement. Process standardization requires further investigation to reduce this health care burden.
\end{abstract}

Key Words: Feeding device, Gastrostomy, Gastrojejunostomy, Hospital resource utilization

\section{INTRODUCTION}

Gastrostomy feeding device (GD) placement is a common pediatric procedure associated with frequent complications and significant hospital resource utilization. ${ }^{[1-3]}$ Most complications are associated with long-term device maintenance and include excess granulation tissue, infection, device dysfunction, peritonitis, pain, and leakage. ${ }^{[4,5]}$ Beyond the risk of complications, each tube requires intensive ongoing care after placement both at home and by medical professionals. Some of this utilization depends on the type of device placed. For example, in many institutions percutaneous endoscopic gastrostomy (PEG) tubes require a planned device change that is scheduled under anesthesia. While the procedure itself may be minor, it requires additional physician time,

*Correspondence: Morgan K Richards; Email: mkr19@u.washington.edu; Address: Division of Pediatric General and Thoracic Surgery, Seattle Children's Hospital, 4800 Sand Point Way NE, Box OA.9.220, 98105, Seattle, Washington, United States. 
nursing resources, and a second anesthetic. As with many institutions, multiple services are responsible for placing GDs. The numerous medical and surgical services involved in the care of children requiring GD insertion make them a heterogeneous and difficult patient population to study. The existing literature examines the outcomes of GD placement in certain patient populations or with a particular method of insertion. ${ }^{[2,4,6-8]}$ While multiple methods for GD insertion have been described, including open, laparoscopic, endoscopic and fluoroscopic approaches, no study to date compares outcomes of all of these methods while controlling for characteristics of pediatric patients with multiple complex medical problems. ${ }^{[1,3]}$

In order to understand the full scope of the problem of high resource utilization, we must first understand the landscape of gastrostomy device placement. The purpose of our study is not to study the complication rate of a single method for placement or the resource utilization of a particular type of patient, but rather to understand what resource utilization is required in the process of device placement and maintenance at the hospital level. We sought to determine, beginning at a single institution, how many devices are placed, what services are involved in placement, and how many returns to the system there are based on specific factors. The factors of interest for this study were the type of device initially placed and the service that performed the placement. While it may be common knowledge that Gastrojejunostomy devices (GJs) require more maintenance than GD's, again the specifics of this difference have never been studied. We examined all reasons for health care utilization, not simply complications, because our experience suggests that the significant amount of health care utilization related to GD placement occurs after device insertion and is a formidable strain on the health care system and the families of these complicated patients. An important benefit to practicing clinicians will be to be able to prepare patients and their families for the challenges they may face after placement of planned or unplanned interventions. Enhanced parental education may help reduce both the burden on the health care system and the strain on families.

\section{MethodS}

A retrospective review was performed of all patients who received a new GD or GJ at our institution between January 1, 2010 and December 31, 2011, after approval by the institutional review board (approved application \#14816). The data were initially obtained using internal administrative data and verified using chart review. Patients were excluded if the procedure was performed at another institution or if a fundoplication was performed at the time of device placement.

Published by Sciedu Press
Demographic information including age, gender, American Society of Anesthesiologists (ASA) Physical Status, and up to nine pre-existing comorbidities based on ICD-9-CM diagnostic codes found listed in the patient's chart were collected for each patient. Characteristics of the device placement were documented, including operative provider specialty and the type of device inserted (GD or GJ). Three different methods for placement were identified for initial device placement: operative, endoscopic, or image-guided placement. Operative placement was performed with either the open, laparoscopic or robotic approach under general anesthesia. Endoscopic placement was performed jointly with a gastroenterologist to provide the esophago gastroduo denoscopy (EGD) for insertion point localization and a surgeon to perform the puncture and PEG placement under general anesthesia. Interventional radiologists inserted the device under image-guidance under general anesthesia.

The primary outcome of interest was the requirement for gastrostomy-related procedures or emergency department visits following initial placement. For each patient that was identified as having primary GD or GJ placement during the study period, the chart was reviewed for any operative, endoscopic, or fluoroscopic imaging procedures related to the device. All emergency department contacts related to the GD or GJ were also recorded. Planned and unplanned gastrostomy-related procedures included 1) device changes or replacements performed in the operating room, gastroenterology suite, or using fluoroscopic guidance, 2) GD or GJ location verification using fluoroscopy following inadvertent removal and subsequent replacement, 3) successful or attempted conversion of a GD to a GJ or a GJ to a GD, 4) GD or GJ removal in the operating room, and 5) gastrocutaneous fistula closure.

Reasons for visits to the emergency department included problems related to the surgical site, the device itself, or feeding. Issues with the gastrostomy site included infection, pain, bleeding, and excess granulation tissue. Complaints relating to the device itself included device displacement or placement concerns, clogged tubing, leaking or broken devices. Feeding difficulties included abdominal distention, oral intake intolerance, and emesis. Exposures of interest included the type of device initially placed (GD or GJ) and the method for initial placement (operative, endoscopic or image-guided).

Univariate and bivariate analyses were performed to describe the population. Two multivariate models were employed to assess resource utilization related to feeding device placement. The first model was an adjusted Poisson regression, which was utilized to estimate the association between ad- 
ditional interventions or GD related emergency department visits and the type of device inserted at initial placement. Poisson regression was selected for its ability to estimate the rate of utilization per person-time associated with the type of device initially placed. The independent variable was the type of device, GT versus GJ at initial placement. The dependent variable was additional hospital utilization defined as GD related emergency department visits or repeat interventions. Confounders in this model included age, gender, ASA class, patient comorbidities, and the specialty of the operative provider, which were incorporated as covariables (see Equation 1). The second model was an adjusted multivariate Cox Proportional Hazards Regression, which was used to calculate the association between method of initial device placement and time to first repeat hospital utilization event. The independent variable was the method for device placement and included surgical, endoscopic or image-guided techniques. The dependent variable of interest was the time to first intervention or emergency department visit. Confounders that were controlled for as covariables included age, gender, initial type of device placed, ASA class and patient (see Equation 2). Statistical significance was set at the .05 level. The analysis was performed using StataCorp. 2011. Stata Statistical Software: Release 12. College Station, TX: StataCorp LP.

$$
\begin{aligned}
& \text { Model } 1: \text { PoissonRegression : Resource Utilization }=2.05 \times \text { GT vs. GJ }+1.00 \times \text { age }+0.82 \times \text { gender }+0.90 \\
& \times \text { ASAClass }+1.29 \times \text { Methodof Placement }+2.01 \times \text { Genetic }+1.04 \times \text { Hematology } / \text { Oncology }+1.09 \times \\
& \text { Cardiac }+0.90 \times \text { Pulmonary }+1.50 \times \text { Renal }+1.36 \times \text { GI }+0.91 \times \text { Other Comorbidity }
\end{aligned}
$$

$$
\begin{aligned}
& \text { Model } 2: \text { CoxProportionalHazardRatio }: \text { Resource Utilization }=2.56 \times \text { Endoscopic vs. Surgical }+2.07 \times \\
& \text { Image }- \text { guided vs. Surgical }+1.00 \times \text { age }+1.01 \times \text { gender }+1.98 \times \text { GTvs.GJ }+0.83 \times \text { ASAClass }+1.22 \times \\
& \text { Genetic }+1.53 \times \text { Hematology } / \text { Oncology }+0.94 \times \text { Cardiac }+1.05 \times \text { Pulmonary }+0.78 \times \text { Renal }+1.44 \times G I \\
& +1.21 \times \text { Other Comorbidity }
\end{aligned}
$$

In equation 1 and 2, GT: Gastrostomy tube; GJ: Gastrojejunostomy; Method of Placement: surgical, endoscopic, image-guided; Genetic: Genetic or neurologic comorbidity; Hematology/Oncology: Hematologic/Oncologic comorbidity; Cardiac: Cardiac comorbidity; Pulmonary: Pulmonary comorbidity; Renal: Renal comorbidity; GI: Gastroenterological comorbidity

\section{RESULTS}

\subsection{Demographics}

A total of 299 children underwent initial GD or GJ placement over the two-year study period. Of those, 275 (92.0\%) were GDs. The ages at initial placement ranged from birth to 22 years (mean 2.47 years, standard deviation 4.32 years). Just under half $(146,48.8 \%)$ were female. More than half of the patients were designated as ASA Class III $(175,58.5 \%)$. The most frequently noted associated comorbidity was genetic anomaly, which included genetic and neurologic impairments $(263,88.0 \%)$ (see Table 1$)$

\subsection{Outcomes}

Fifty-four percent of initial GDs placed were in male patients compared to $20.8 \%$ of GJs. One hundred ninety one $(69.5 \%)$ GDs were placed in patients with an ASA Class III or IV compared to 22 (91.7\%) of GJs. Of the GDs placed, 182
(60.9\%) patients had at least one additional encounter related to their feeding device. Forty percent of GDs had a single encounter that consisted of device placement, compared to $25 \%$ of GJs. Using Poisson regression, after controlling for the age, gender, ASA Class, and provider specialty, initial GJ placement was associated with 2.05-fold increased rate of additional hospital resource utilization compared to GD placement (95\% CI: 1.01 - 4.16) (see Table 2).

A majority of the devices were initially placed operatively (145, 48.5\%), followed by endoscopic placement (113, $37.8 \%)$, and lastly the image-guided technique $(41,13.7 \%)$. Most patients who underwent device placement were under the age of two including $77.9 \%$ of patients with operative placement, $73.2 \%$ of patients with image-guided placement and $52.2 \%$ of patients with endoscopic placement. Most operative and image-guided patients had an ASA Class of III (60.0\% and 68.3\% respectively) compared to endoscopic placement where most were ASA Class I (75.0\%).

Many devices required repeat utilization following placement. Thirty-three of $41(80.5 \%)$ of those placed by the image-guided technique required repeat utilization as compared to 84/113 (74.3\%) placed endoscopically and 56/145 (44.8\%) placed operatively (see Figure 1). After initial de- 
vice placement, there were 283 repeat interventions and 103 ER visits (see Figure 2). A Cox Proportional-Hazards Regression controlling for age, gender, ASA Class, patient comorbidities, and type of device placed estimated that endoscopic compared to operative placement was associated with a hazard ratio for repeat hospital utilization of $2.56(95 \%$ CI: 1.73 - 3.79) and image-guided compared to operative placement was associated with a hazard ratio of 2.07 (95\% CI: 1.20 - 3.58) (see Table 3). A Kaplan-Meier survival curve shows the time to first event after placement stratified by operative provider and adjusted for patient characteristics including age, gender, ASA Class, patient comorbidities and type of device placed (see Figure 3).

Table 1. Demographic and health characteristics of patients undergoing gastrostomy or gastrojejunostomy device placement and subsequent hospital utilization

\begin{tabular}{|c|c|c|c|c|}
\hline & Repeat Utilization N (\%) & No Repeat Utilization N (\%) & Total Population N (\%) & $p$-value \\
\hline Overall & $182(60.9)$ & $117(39.1)$ & $299(100)$ & \\
\hline Female & $88(60.3)$ & $58(39.7)$ & $146(48.8)$ & .8 \\
\hline Age, years (mean, $S D$ ) & $3.06(4.50)$ & $2.78(4.06)$ & $2.95(4.33)$ & .9 \\
\hline ASA Class & & & & .4 \\
\hline $\mathrm{I}$ & $3(75.0)$ & $1(25.0)$ & $4(1.3)$ & \\
\hline II & $53(64.6)$ & $29(35.4)$ & $82(27.4)$ & \\
\hline III & $107(61.1)$ & 68 (38.9) & $175(58.5)$ & \\
\hline IV & $19(50.0)$ & $19(50.0)$ & $38(12.7)$ & \\
\hline Type of Device & & & & .1 \\
\hline Gastrostomy & $164(59.6)$ & $111(37.1)$ & $275(92.0)$ & \\
\hline Gastrojejunostomy & $18(75.0)$ & $6(2.0)$ & $24(8.0)$ & \\
\hline \multicolumn{5}{|l|}{ Comorbidities* } \\
\hline Cardiac & $61(56.5)$ & $47(43.5)$ & $108(36.1)$ & .2 \\
\hline Pulmonary & 78 (61.9) & $48(38.1)$ & $126(42.1)$ & .8 \\
\hline Hematology/Oncology & $27(64.3)$ & $15(35.7)$ & $42(14.0)$ & .6 \\
\hline Genetic Anomaly & $164(62.4)$ & 99 (37.6) & $263(88.0)$ & .2 \\
\hline Renal & 18 (52.9) & $16(47.1)$ & $34(11.4)$ & .3 \\
\hline Acquired Gastrointestinal & $115(65.0)$ & $62(35.0)$ & $177(59.2)$ & .1 \\
\hline Other & $90(61.2)$ & $57(38.8)$ & $147(49.2)$ & .9 \\
\hline
\end{tabular}

* Patients may have multiple comorbidities

Table 2. Multivariate Poisson Regression of the incident rate ratio of repeat intervention or emergency department visit associated with initial gastrojejunostomy relative to gastrostomy device placement

\begin{tabular}{llll}
\hline & IRR $^{*}$ & $\mathbf{9 5 \%} \mathbf{C I}^{*}$ & $\boldsymbol{p}_{\text {-value }}{ }^{* *}$ \\
\hline GJ versus GD* & 2.05 & $1.01-4.16$ & .046 \\
Age & 1.00 & $1.00-1.00$ & .2 \\
Gender & 0.82 & $0.61-1.10$ & .2 \\
ASA Class & 0.90 & $0.71-1.13$ & .4 \\
Provider Specialty & 1.29 & $0.94-1.76$ & .1 \\
Comorbidities & & & \\
Cardiac & 1.09 & $0.81-1.47$ & .6 \\
Pulmonary & 0.90 & $0.67-1.20$ & .5 \\
Hematology/Oncology & 1.04 & $0.64-1.69$ & .9 \\
Genetic Anomaly & 2.01 & $1.26-3.19$ & .003 \\
Renal & 1.50 & $0.85-2.64$ & .2 \\
Acquired Gastrointestinal & 1.36 & $0.98-1.90$ & .07 \\
Other & 0.91 & $0.66-1.25$ & .6 \\
\hline
\end{tabular}

"IRR: Incident rate ratio; 95\% CI: 95\% Confidence Interval; GJ: gastrojejunostomy; GD: gastrostomy device; ASA Class: American Society of Anesthesiologists physical status classification; ${ }^{* *} p$-value $<.05$ was considered statistically significant

Published by Sciedu Press

\section{Discussion}

Our data suggest that endoscopic and image-guided techniques for initial feeding device placement are associated with higher rates of hospital resource utilization than operative placement. Additionally, GJs are significantly more likely to require repeat utilization than GDs. These differences remain significant even when controlling for confounding factors. Patients requiring GD or GJ placement frequently have multiple comorbidities.

The method for initial device placement is associated with different rates of hospital resource utilization. In our health care system younger patients and patients with higher ASA Class tended to have their device placed either operatively or using the image-guided technique compared to endoscopic placement. The association may be related to the method itself and the differences in required follow-up. Some of the higher utilization observed might be related to the fact that endoscopic and image-guided devices have scheduled 
initial and subsequent exchanges such as a PEG to button, whereas surgically placed GDs require a single procedure for placement.

Table 3. Multivariate Cox Regression of hazard ratio of repeat intervention or emergency department visit associated with the method of initial placement

\begin{tabular}{llll}
\hline & HR $^{*}$ & $\mathbf{9 5 \%} \mathbf{C I}^{*}$ & p-value $^{* * *}$ \\
\hline Method of placement & & & \\
Endoscopic versus Operative & 2.56 & $1.73-3.79$ & $<.001$ \\
Image-guided versus Operative & 2.07 & $1.20-3.58$ & .009 \\
Age & 1.00 & $1.00-1.00$ & .3 \\
Gender & 1.00 & $0.75-1.36$ & 1.0 \\
GJ versus GD* & 1.98 & $0.98-3.99$ & .06 \\
ASA Class & 0.83 & $0.64-1.07$ & .2 \\
Comorbidities & & & .8 \\
Cardiac & 0.94 & $0.65-1.37$ & .8 \\
Pulmonary & 1.05 & $0.77-1.43$ & .8 \\
Hematology/Oncology & 1.53 & $1.01-2.34$ & .047 \\
Genetic Anomaly & 1.22 & $0.74-2.00$ & .4 \\
Renal & 0.78 & $0.47-1.30$ & .3 \\
Acquired Gastrointestinal & 1.44 & $1.04-1.99$ & .03 \\
Other & 1.21 & $0.87-1.67$ & .3 \\
\hline IRR: Inciden ras & & & \\
\hline
\end{tabular}

"IRR: Incident rate ratio; 95\% CI: 95\% Confidence Interval; GJ: gastrojejunostomy; GD: gastrostomy device; ASA Class: American Society of Anesthesiologists physical status classification; ${ }^{* *} p$-value $<.05$ was considered statistically significant

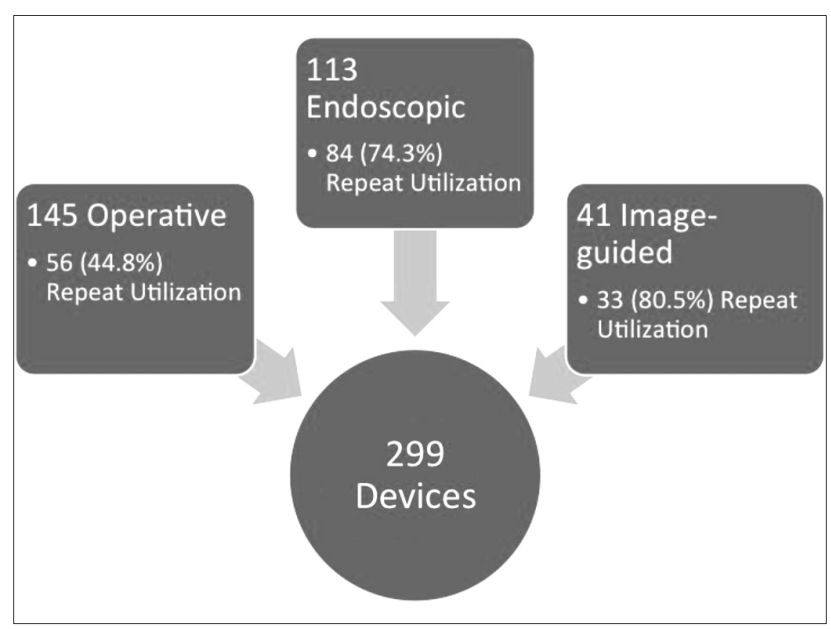

Figure 1. Methods of feeding device placement and number requiring additional hospital utilization

Our data corroborate the findings that GDs and GJs are associated with multiple interventions and emergency department visits. ${ }^{[4,5]}$ Although patients who require feeding devices often have high health care utilization for their ongoing needs, this relatively simple procedure appears to add to that burden. There may be many potential explanations for the association between hospital resource utilization and GJ relative to GD placement. For example, GJs require increased techni- cal complexity for placement or replacement. A GD with a well-formed track may be replaced at home or in clinic, while GJ replacement requires fluoroscopic technique to verify placement. The device is more complex and thus has more potential for dysfunction, including device occlusion and improper migration back into the stomach. The association between increased hospital resource utilization and GJ placement is important when counseling parents about life with a feeding device as it may better prepare them for their child's long-term clinical course.

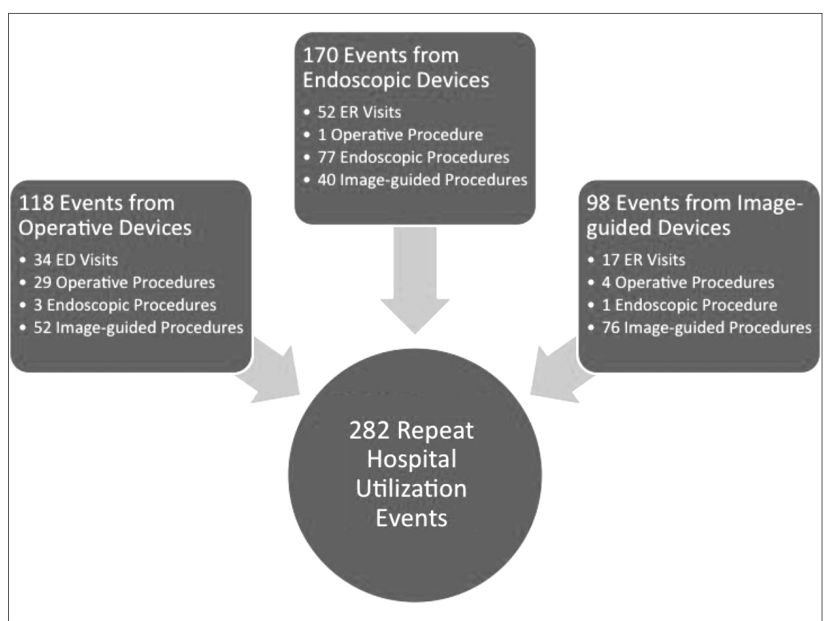

Figure 2. Flow diagram of the number of additional utilization events by method of initial placement

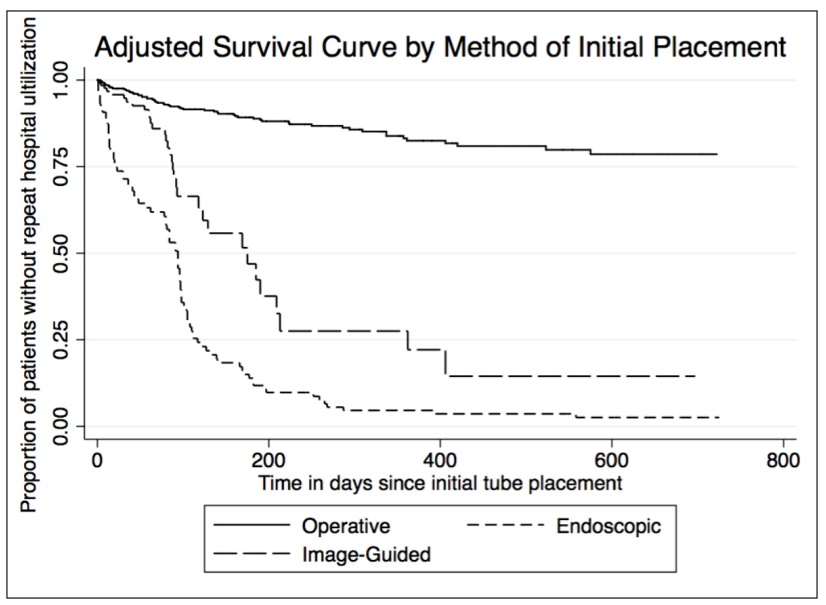

Figure 3. Adjusted survival curve by method of initial placement

Although this is one of the largest and most diverse patient cohorts of GDs and GJs to date, there are limitations to the study. It is retrospective, therefore, association but not causation may be inferred. Since it is nonrandomized, there may be residual confounding. This study captures not only GD-related complications or unplanned events, but all GD- 
related interventions, including planned procedures, with the intent of highlighting resource utilization overall. As mentioned previously, the indication for the feeding device was not available in detail, thus limiting the utility of this information when stratifying patients or controlling for confounding. Finally, type of device placed is not consistently recorded in the operative and procedural notes so we were not able to stratify by this factor, although we were able to stratify by the initial method of placement (operative, endoscopic or image-guided).

Despite these limitations, this study provides valuable information about the differences in outcomes based on the type of device placed and the method for initial device placement. We are now able to counsel patients regarding their risk of further intervention, both planned and unplanned, prior to device placement based on the type of device and the method of placement. Additionally, this allows for system level quality improvement projects to determine if the planned procedures are required. A pre-procedure checklist will likely be useful for ensuring that patients have proper workups and receive the correct device at initial placement. Now that we have a sense of overall hospital utilization, we can embark on qual- ity improvement measures to reduce this significant burden on families and the health care system as a whole.

Hospital resource utilization is dependent on the method of initial device placement and whether a gastrostomy or gastrojejunostomy are used at initial placement. Patients are more likely to require additional hospital resources if they receive a GJ at initial placement or if the placement occurs using the endoscopic or image-guided technique. In order to address the differences in utilization by type of device and method used, a protocol has been established at our institution that helps to identify those patients that require gastrostomy placement and identify a care team to admit the patient following the procedure. The goal of the protocol is to minimize the length of stay, cost, patient discomfort, duration of anesthesia, and radiation exposure. The feeding advancement schedule has also been standardized in an effort to reduce variation in practice patterns. Now that this study has established the overall hospital utilization and defined areas of improvement, future directions include a comparative study examining the difference in resource utilization between patients treated prior to and after protocol implementation.

\section{REFERENCES}

[1] Friedman JN, Ahmed S, Connolly B, et al. Complications associated with image-guided gastrostomy and gastrojejunostomy tubes in children. Pediatrics. 2004; 114(2): 458-461. PMid: 15286230. http://dx.doi.org/10.1542/peds.114.2.458

[2] Baker L, Emil S, Baird R. A comparison of techniques for laparoscopic gastrostomy placement in children. J Surg Res. 2013; 184(1): 392-396. http://dx.doi .org/10.1016/j.jss.2013.05.067

[3] Wu TC. Percutaneous endoscopic gastrostomy in children-issues remain. Pediatr Neonatol. 2013; 54(5): 291-292. http://dx.doi .org/10.1016/j.pedneo.2013.06.002

[4] Fascetti-Leon F, Gamba P, Dall'Oglio L, et al. Complications of percutaneous endoscopic gastrostomy in children: results of an Italian multicenter observational study. Dig Liver Dis. 2012; 44(8): 655-659. http://dx.doi.org/10.1016/j.dld.2012.03.017
[5] Ackroyd R, Saincher M, Cheng S, et al. Gastrostomy tube insertion in children: the Edmonton experience. Can J Gastroenterol. 2011; 25(5): 265-268.

[6] Sy K, Dipchand A, Atenafu E, et al. Safety and effectiveness of radiologic percutaneous gastrostomy and gastro jejunostomy in children with cardiac disease. AJR Am J Roentgenol. 2008; 191(4): 1169-1174. http://dx.doi.org/10.2214/AJR.07.3655

[7] Bradley GM, Carson KA, Leonard AR, et al. Nutritional outcomes following gastrostomy in children with cystic fibrosis. Pediatr Pulmonol. 2012; 47(8): 743-748. http://dx.doi.org/10.1002/p pul. 22507

[8] Brewster BD, Weil BR, Ladd AP. Prospective determination of percutaneous endoscopic gastrostomy complication rates in children: still a safe procedure. Surgery. 2012; 152(4): 714-9- discussion 719-21. http://dx.doi.org/10.1016/j.surg.2012.07.018 